\title{
Proof-of-Principle Direct Measurement of Landau Damping Strength at the Large Hadron Collider with an Antidamper
}

\author{
S. A. Antipov $\odot,{ }^{1,2, *}$ D. Amorim $\odot,{ }^{1,3}$ N. Biancacci, ${ }^{1}$ X. Buffat, ${ }^{1}$ E. Métral $\odot,{ }^{1}$ \\ N. Mounet, ${ }^{1}$ A. Oeftiger ${ }^{1,4}$ and D. Valuch ${ }^{1}{ }^{1}$ \\ ${ }^{1}$ European Organization for Nuclear Research (CERN), Geneva 1211, Switzerland \\ ${ }^{2}$ Deutsches Elektronen-Synchrotron (DESY), Hamburg 20459, Germany \\ ${ }^{3}$ Synchrotron SOLEIL, Gif-sur-Yvette 91192, France \\ ${ }^{4}$ Helmholtzzentrum für Schwerionenforschung (GSI), Darmstadt 64291, Germany
}

(Received 7 August 2020; revised 13 January 2021; accepted 12 March 2021; published 20 April 2021)

Landau damping is an essential mechanism for ensuring collective beam stability in particle accelerators. Precise knowledge of the strength of Landau damping is key to making accurate predictions on beam stability for state-of-the-art high-energy colliders. In this Letter, we demonstrate an experimental procedure that would allow quantifying the strength of Landau damping and the limits of beam stability using an active transverse feedback as a controllable source of beam coupling impedance. In a proof-of-principle test performed at the Large Hadron Collider, stability diagrams for a range of Landau octupole strengths have been measured. In the future, the procedure could become an accurate way of measuring stability diagrams throughout the machine cycle.

DOI: 10.1103/PhysRevLett.126.164801

Landau damping (LD) of collective oscillation modes in collisionless plasmas, where particles interact via long-range forces, was originally predicted in Ref. [1] and later observed in Ref. [2]. In particle accelerators, LD arises from a natural spread of synchrotron and betatron frequencies of particles within the beam. It is crucial for stabilization of intense beams, which would otherwise suffer from various kinds of collective unstable modes that may strongly degrade the beam quality, both in the longitudinal [3] and in the transverse degrees of freedom [4]. Precise knowledge of the strength of Landau damping is key to making accurate predictions on beam stability for high-energy colliders such as the Large Hadron Collider (LHC) [5,6] or its High-Luminosity upgrade [7] as well as future machines such as the Future Circular Collider with its hadron version (FCC-hh) [8]. Other examples are the intermediate-energy hadron machines running at high intensities such as the FAIR synchrotron SIS100, which rely on Landau damping to suppress beam instabilities $[9,10]$.

Typically, Landau damping in particle accelerators is approached via stability diagram (SD) theory [11,12]. Given the complex frequency of collective motion $\Delta \omega$, the frequency in the presence of $\operatorname{LD} \Omega$ is found by the generic dispersion relation (here, for a horizontal, $x$ plane):

Published by the American Physical Society under the terms of the Creative Commons Attribution 4.0 International license. Further distribution of this work must maintain attribution to the author(s) and the published article's title, journal citation, and DOI.

$$
\Delta \omega=-1 / \int \frac{J_{x} \partial F / \partial J_{x}}{\Omega+\delta \omega\left(J_{x}, J_{y}\right)+i o} d J_{x} d J_{y}
$$

where $F\left(J_{x}, J_{y}\right)$ is the beam unperturbed distribution function in action-angle phase space $(J, \theta), \delta \omega$ the action-dependent frequency shift, and io a vanishingly small imaginary number added to the integration path. In general, to find $\Omega$, one has to solve Eq. (1) for all $\Delta \omega$. The $\mathrm{SD}$ is defined as the threshold of stability, i.e., a contour in $\Delta \omega$ space where $\operatorname{Im} \Omega=0$. Figure 1 shows an example SD for LHC at $450 \mathrm{GeV}$ with $\Delta \omega$ normalized by the synchrotron frequency $\omega_{s}$.

A common technique of measuring SDs is by means of beam transfer function (BTF) measurements [13] through frequency dependence of the response to forced beam oscillations. BTF has been successfully used to measure stability diagrams at GSI [14] and RHIC [15] and at injection energy in LHC [16]. The method has some limitations though: It is challenging to maintain both good beam stability and high signal-to-noise ratio when driving the oscillation [17]. Most importantly, the measurement does not test the strength of Landau damping itself but the transfer function instead, relying on multiple assumptions behind Eq. (1): The synchrotron frequency spread is negligible, the betatron frequency spread is sufficiently small, the beam response to an external excitation is linear, and the coherent modes are uncoupled.

We propose a new alternative approach for measuring the strength of LD using a transverse feedback system. Feedbacks are widely used in particle accelerators to 
modify and study beam dynamics [18-23]. High-intensity machines, in particular, rely on them to damp beam centroid oscillations by measuring the centroid of individual bunches and kicking them in counterphase [24]. By reverting the polarity of the transverse feedback, one can excite a collective mode in the beam, thus creating an antidamper that amplifies coherent oscillation modes instead of suppressing them. The antidamper then acts as a controllable source of beam coupling impedance. Thus, observing at which feedback gain the beam becomes unstable, one obtains a direct measurement of the strength of LD in the synchrotron. Furthermore, with an accurate control over the feedback phase, one can explore the full complex plane of tune shift and growth rate, obtaining a SD for comparison with theoretical predictions or tracking simulations. In this Letter, we describe a proof-of-principle test to measure the strength of LD created by the LHC octupole system at $450 \mathrm{GeV}$ injection energy.

Consider a relativistic beam in an accelerator with a revolution period $T_{0}$, single-particle transverse and longitudinal angular frequencies $\omega_{\beta}$ and $\omega_{s}$, respectively, a normalized chromaticity $\xi=\left(1 / \omega_{\beta}\right)\left(d \omega_{\beta} / d \delta_{p}\right)$ (with $\delta_{p}$ the relative deviation of the longitudinal momentum with respect to the design value), and a phase slip factor $\eta=\left(1 / T_{0}\right)\left(d T_{0} / d \delta_{p}\right)$. Assuming a small, horizontal perturbation of the distribution $F$, a coherent transverse oscillation mode can be described as

$$
\Delta F=f\left(J_{x}\right) e^{i \theta_{x}} g\left(J_{s}\right) e^{i l \theta_{s}} e^{i \xi \omega_{\beta} s / \eta c} e^{-i \omega s / c},
$$

where functions $f$ and $g$ describe its transverse and longitudinal structure, respectively. $l$ is an integer azimuthal mode number, with $l=0$ corresponding to a dipole, or c.m. oscillation. The last term governs the mode's temporal structure with $\omega=\omega_{\beta}+l \omega_{s}+\Delta \omega$, where $\Delta \omega$ is a small complex mode frequency shift, resulting from the interaction of the beam with its self-wakefields. The middle term contains the head-tail phase advance $\chi=\xi \omega_{\beta} s / \eta c$.

If the variation of the feedback's dynamic response over the bunch length can be neglected, it can be described as a constant wake force acting on the beam $W(z)=W_{0}=$ const, which corresponds to a $\delta$-functionlike coupling impedance $[25,26] Z_{d}(\omega) \propto g \times e^{i \phi} \times \delta(\omega)$, where $g$ stands for feedback gain and $\phi$ for its phase: 0 for a resistive feedback (picking up on beam position) and $90^{\circ}$ for a reactive one (picking up on transverse beam momentum). Such impedance shifts the frequencies of collective modes by $\Delta \omega \propto-i \times g \times e^{i \phi}$. A resistive feedback, thus, moves a coherent beam mode upward in the diagram, driving it unstable, with a growth rate of $-g$. Such an antidamper with $\phi=0$ has been proposed for the IOTA ring $[27,28]$.

The LHC transverse feedback $[29,30]$ consists of beam position measurement units, digital signal processing units, and a set of power amplifiers feeding electrostatic deflectors and utilizes an adjustable digital phase shifter to compensate the phase advance between the pickups and the kickers (Fig. 1). It is capable of independently adjusting the gain and the phase delay between the pickups and the kickers, thus setting the feedback transfer function anywhere in the complex plane $g \times e^{i \phi}$ and allowing operation as a source of controlled beam impedance. This "impedance" can be instantly changed between two consecutive revolutions during the beam abort gap.

The feedback stripline pickups measure beam position every turn, and the sum $(\Sigma)$ and difference $(\Delta)$ signals are calculated by an analog hybrid coupler. These rf signals are quadrature demodulated to baseband I-Q pairs and digitized by 16-bit analog-to-digital converters. Next, the bunch position is normalized to remove the dependency on bunch intensity. The turn-by-turn data stream is then split between the transverse feedback function and an external observation system which provides the digital position data at full rate [31]. Finally, a real time monitor calculates the amplitude of the transverse motion and compares it with a predefined threshold for an automated instability detection. The system can measure the beam position with a full scale of $2 \mathrm{~mm}$; its noise floor with a single pickup is $2 \mu \mathrm{m}$.

A proof-of-principle test has been performed with single pilot bunches of $0.5 \times 10^{10} \mathrm{p}$ in $1 \mu \mathrm{m}$ normalized $\mathrm{rms}$ emittance (Table I). These bunches are not scraped transversely and are expected to have a close to normal transverse profile [32]. At this intensity and the nominal ring chromaticity of $Q^{\prime}=\left(\omega_{\beta} / 2 \pi\right) \xi=14$ (rms head-tail phase $\chi=0.2)$, the dipolar azimuthal head-tail mode (a)

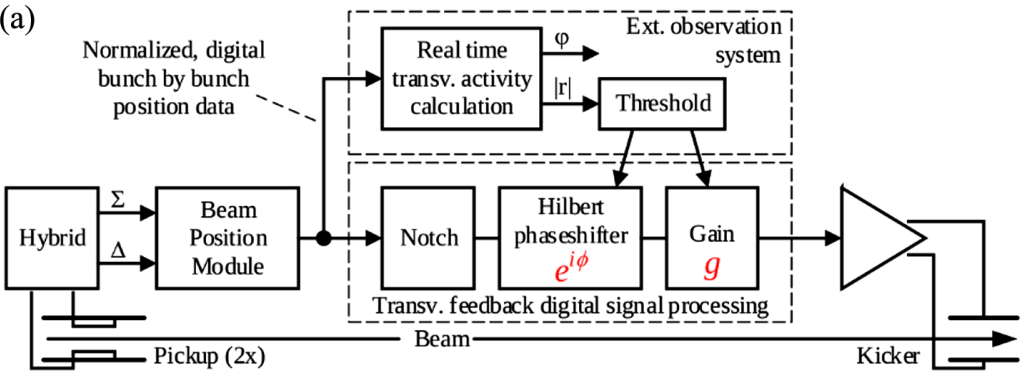

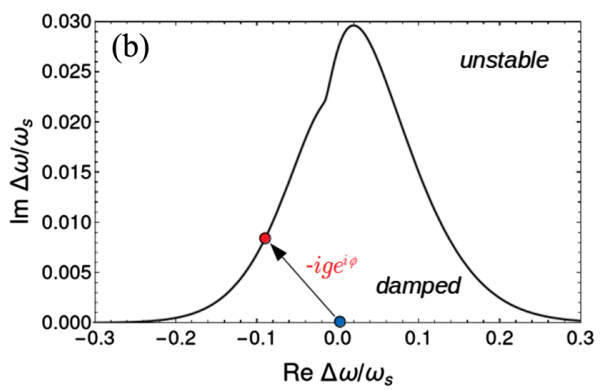

FIG. 1. Schematic of the LHC transverse feedback system (a) and its impact on a collective mode within the LHC stability diagram (b) for a $450 \mathrm{GeV}$ Gaussian beam with $4 \times 10^{-5} \mathrm{rms}$ tune spread generated by the LHC octupole system. 
TABLE I. Key parameters used for the study.

\begin{tabular}{lclc}
\hline \hline Bunch energy & $450 \mathrm{GeV}$ & rf voltage & $6 \mathrm{MV}$ \\
Bunch intensity & $5 \times 10^{9} \mathrm{p}$ & Synchrotron freq., $\omega_{s}$ & $0.03 \mathrm{~s}^{-1}$ \\
Emittance, rms & $1.0-1.1 \mu \mathrm{m}$ & Chromaticity & 14 \\
Bunch length, rms & $0.25 \mathrm{~ns}$ & H.-t. phase, rms, $\chi$ & 0.02 \\
Revolution period & $89 \mu \mathrm{s}$ & Linear coupling, $\left|C^{-}\right|$ & $10^{-3}$ \\
\hline \hline
\end{tabular}

typically dominates the landscape with higher-order modes featuring much weaker growth rates, and the mode shift from beam coupling impedance as well as reducing space charge (SC) effects is minimized: The SC tune shift is around $10^{-4}$. At the same time, the low energy allows applying a large octupolar detuning to stabilize the beam without the transverse feedback.

In the LHC, the betatron frequency spread required to produce the LD is largely generated by a dedicated system of superconducting octupole magnets [5]. The frequency shift can be considered linear: $\delta \omega=a J_{x}+b J_{y}$, where $a$ and $b$ are constants proportional to the octupole current. A set of measurements was performed with LD produced by several configurations of relatively high octupole currents: $I_{\text {oct }}=11$ and $17 \mathrm{~A}$, which correspond to roughly $\sigma_{Q}=4$ and $7 \times 10^{-5} \mathrm{rms}$ tune spread, respectively, and with the octupole system off, when machine nonlinearities create $\sigma_{Q}=10^{-5}$. Also, one measurement was done at a negative octupole current $I_{\text {oct }}=-11 \mathrm{~A}$, when the signs of $a$ and $b$ are inverted and the resulting SD is a mirror image of that for $11 \mathrm{~A}$, reflected around the $\operatorname{Re} \Delta \omega=0$ line. Additionally, the measurement at $\sigma_{Q}=4 \times 10^{-5}$ was repeated at a low chromaticity of $Q^{\prime}=3(\chi=0.05)$.

First, in order to confirm the feedback was acting as a controlled source of impedance, the measurement was performed at three antidamper phases: $0,45^{\circ}$, and $65.7^{\circ}$ with the octupole system off and the betatron tune spread produced only by machine nonlinearities. At each phase, the feedback excited a beam instability, and the growth rate of the c.m. oscillations was measured as a function of the feedback gain. The instability detection threshold was set at $200 \mu \mathrm{m}$ - a value comparable to the rms transverse size of the beam and 100 times greater than the feedback noise floor of $2 \mu \mathrm{m}$. This threshold allowed capturing almost five growth time constants and observing an onset of the instability at an early stage. The data were acquired from the feedback pickups via its digital observation system using a 64000 -turn data buffer; an example of an acquisition is presented in Supplemental Material [33]. The observation system extracted the growth rate from the positional data time series using a Hilbert transform to calculate the oscillation amplitude turn by turn and exponential fitting [34]. The resulting dependence of the instability growth rate on the feedback gain was found to be linear (Fig. 2).

The experimental findings were then compared to sixdimensional macroparticle tracking in PyHEADTAIL $[35,36]$, including SC that can affect the spread of betatron frequencies and, thus, the damping when the lattice nonlinearities are small. The code utilizes a smooth optics approximation and a drift-kick model for nonlinear synchrotron motion. Nonlinear effects such as chromatic and octupolar amplitude detuning are applied as effective tune shifts for each individual macroparticle. Collective effects, arising from impedance, space charge, or external feedback are applied at a series of interaction points where the beam is longitudinally divided into a set of slices via a $1 \mathrm{D}$ particle-in-cell (PIC) algorithm.

The natural machine nonlinearities were modeled by an equivalent octupole linear amplitude detuning. The numerical model also included nonlinear longitudinal motion inside the rf bucket, while linear coupling effects were neglected, since they are expected to have little effect on beam stability if the coupling is sufficiently well corrected as discussed further. Without space charge, $10^{6}$ macroparticles have been tracked for $10^{6}$ turns. Simulations including self-consistent space charge (via a $2.5 \mathrm{D}$ sliceby-slice PIC algorithm) are based on $3 \times 10^{6}$ macroparticles being tracked during $6 \times 10^{4}$ turns. Tracking results demonstrate that SC significantly affects the instability growth rate for a given gain of the destabilizing feedback, increasing the stable area (Fig. 2). With SC taken into account, the numerical results are in good agreement with the experimental observations.

The dependence of the instability growth rate on the feedback strength gives an insight into the strength of LD by natural nonlinearities of the lattice, which can be seen as the intersection of the lines with the horizontal axis. These are the points where the beam as a dynamical system is exactly at the limit of stability. For a resistive feedback, an excitation rate of about $2.5 \times 10^{-4} \mathrm{turn}^{-1}$ is needed to

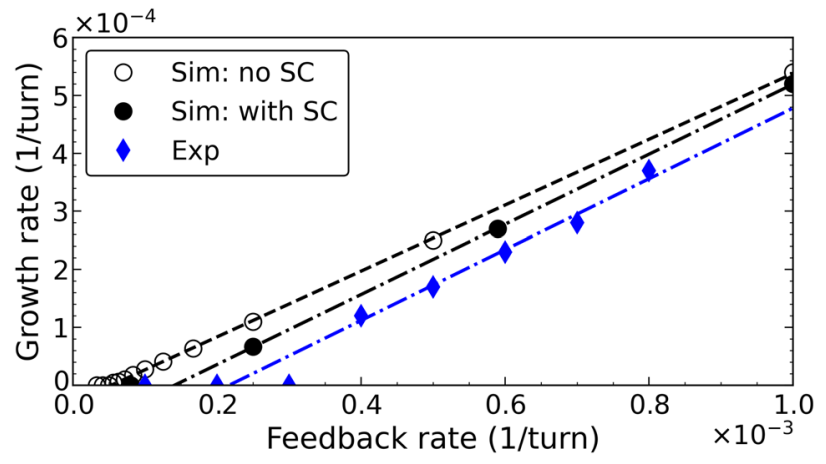

FIG. 2. The instability growth rate scales linearly with the damper gain, allowing one to calibrate the feedback strength. The nonzero gain required to start an instability is caused by natural nonlinearities of the machine. Overall, experimental data (diamonds) are in good agreement with numerical simulations with SC taken into account (filled circles). For comparison, the simulation data for the no-SC case is also shown (empty circles). Dashed and dash-dotted lines represent linear fits of the data above the instability threshold. 


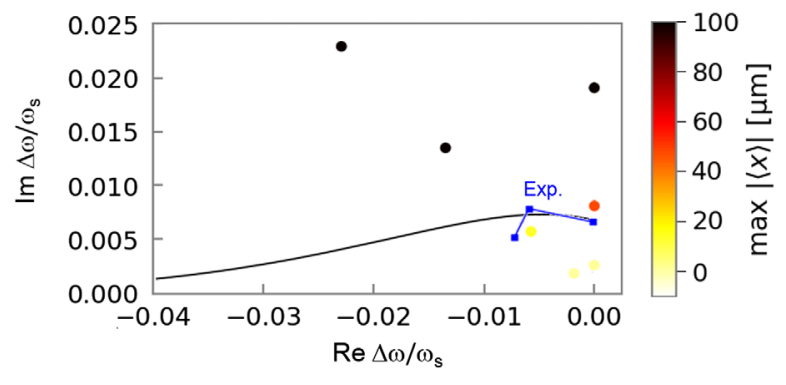

FIG. 3. Stability region observed in particle tracking for the assumed natural nonlinearities in the LHC $(E=450 \mathrm{GeV}$, rms tune spread $10^{-5}$ ) including space charge: The color of the dots depicts the maximum beam excursion. The blue squares and line show the measured limit of stability; the solid black line shows a SD prediction for a Gaussian beam.

destabilize the beam. The blue line in Fig. 3 shows the corresponding stability diagram found by extrapolating the measurement data points at different phases to $\operatorname{Im} \Omega=0$. This threshold resembles the curve expected from a SD theory (shown in black); also, in the tracking with SC, particle amplitudes (colored dots in Fig. 3) start growing above the measured threshold.

For the high octupole current strengths, we performed a series of measurements at different octupole settings to sketch the corresponding SDs, gradually increasing the feedback gain at a given phase until reaching the limit of stability. After an instability had been detected (beam centroid excursion exceeding the threshold of $200 \mu \mathrm{m}$ ), the feedback was automatically switched back to a resistive stabilizing mode with a damping time of 200 turns to avoid further amplitude growth. The gain ramp was performed sufficiently fast to exclude potential impact of latency effects, observed in the LHC on timescales of several minutes that are supposedly related to a hardware noise modifying the bunch distribution [37]. Injection-toinjection spread of the strength of $L D$, measured over five consecutive injections at $4 \times 10^{-5} \mathrm{rms}$ tune spread and $0^{\circ}$ phase-around $7 \%$-indicated a quite good reproducibility of beam distribution. This gives a lower limit on the systematic uncertainty in the measurements.

The shape of the measured SDs qualitatively matches the expectations from a simple linear SD theory. Both the height and the width scale with the octupole current, with the SD for $7 \times 10^{-5} \mathrm{rms}$ tune spread being around $50 \%$ higher than that for $4 \times 10^{-5} \mathrm{rms}$ tune spread, as expected (Fig. 4). The second measurement for $4 \times 10^{-5} \mathrm{rms}$ tune spread made at a lower chromaticity of three units matches within 10\%-20\% the first one performed at 14 units. The negative octupole polarity offers around $30 \%$ greater coverage of the negative tune shifts, also in good qualitative agreement with what one would expect from a diagram of the octupole tune spread. This illustrates why the negative polarity is preferred to suppress impedance-driven instabilities in the LHC that feature negative mode frequency

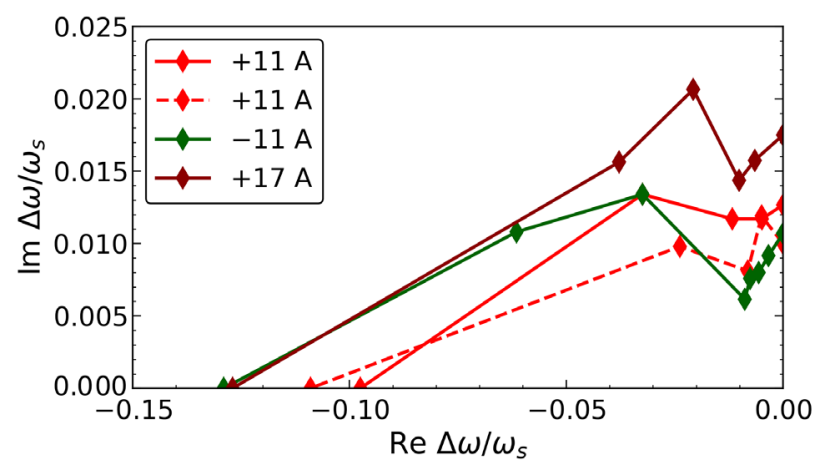

FIG. 4. The measured height of the stability diagrams at $450 \mathrm{GeV}$ scales linearly with the octupole current with the negative octupole polarity providing around $30 \%$ larger coverage of negative mode frequency shifts, which are relevant for coherent beam stability in the LHC. Solid lines, $Q^{\prime}=14$; dashed line, $Q^{\prime}=3$.

shifts. The exact magnitude of the gain should depend on the details of the beam distribution.

A comparison can be made with a series of BTF measurements performed independently over several years at the LHC [17]. The researchers found around factor of 2 discrepancy with the linear SD predictions for octupole currents about 6.5-13 A, similar to the ones used in the present study. The measurement for the tune spread created by natural nonlinearities matched the model. Both these results agree with our findings.

There are several factors that affect the strength of LD and the shape of SDs in the LHC. First, there are several sources of nonlinearities affecting LD at injection energy, ranging from the hysteresis of the octupole correctors to hardware misalignment [38-40], which generate a combined linear detuning with amplitude that is roughly equivalent to $I_{\text {oct }}=-2.5 \mathrm{~A}\left(\sim 10^{-5} \mathrm{rms}\right.$ tune spread $)$.

Second, the linear coupling can reduce the tune footprint locally and as well lead to a large second-order amplitude detuning [40-42]. Hence, in the measurement, the coupling was corrected down to a sufficiently low value of the closest tune approach $\left|C^{-}\right|=10^{-3}$; the resulting reduction of the SD should not have exceeded $10 \%$ for $4 \times 10^{-5} \mathrm{rms}$ tune spread.

Third, the measured stability diagram might be affected by the machine's impedance that provides an extra complex mode frequency shift. According to a simulation in the NHT Vlasov solver, a $50 \%$ higher impedance would be perceived as a $50 \%$ smaller stability diagram generated by the natural machine nonlinearities.

Finally, although space charge on its own does not provide LD for the rigid dipole mode [43], it does modify the SD produced by lattice nonlinearities $[44,45]$. In general, an interplay of octupole detuning and nonlinear space charge may be important as observed in particle tracking simulations [46]. In our tracking simulations, SC even as weak as $\sim 0.1 \omega_{s}$ affects LD and, most importantly, 
the feedback calibration (Fig. 2). Therefore, for a quantitative analysis of the observed SDs, one has to include SC, which is missing in Eq. (1).

In conclusion, in this proof-of-principle test, we have demonstrated that the active feedback system can be used as a source of controlled impedance to probe the strength of Landau damping. The experiment has been carried out in the LHC at injection energy of $450 \mathrm{GeV}$ with single lowintensity bunches. First, the active feedback system has been calibrated to create an arbitrary complex tune shift. Both the tune shift and instability growth rate have been demonstrated to increase linearly with the feedback gain, as expected. Then, the feedback has been utilized to directly measure the strength of LD by gradually increasing its gain until a transverse activity is observed. The possibility of exploring the stability diagram by changing the damper phase has also been demonstrated. A precise quantitative comparison with analytical models requires a good control and an accurate knowledge of beam optics nonlinearities, as well as taking into account the nonlinear space charge force. The results are in good qualitative agreement with the theoretical SD predictions and also in agreement with the BTF measurements, performed independently at the LHC.

The technique has a potential to become a fast nondestructive tool for measuring the strength of LD. Its advantage is that it does not rely on any approximation of the betatron frequency spread or the beam distribution and can, in principle, be used with arbitrary strong beam impedance. It could find applications in studying Landau damping of bunched beams with space charge, where, to the authors' best knowledge, no satisfactory analytical models of LD currently exist and one has to therefore rely on computationally demanding macroparticle simulations. Direct measurements using an antidamper can therefore be of great value for benchmarking such simulations. Finally, in the LHC, the technique would be well suited for studies at the top energy, where the constraints arising from LD are the tightest and the effect of space charge is negligible, and lattice nonlinearities are normally well controlled.

The authors thank Alexey Burov (FNAL) for sharing his ideas which triggered this research. We thank the LHC Operations Team and especially Francesco Velotti for their help carrying out the experiment. We also acknowledge Lukas Malina and Ana García-Tabarés for their insightful tips on data processing and acquisition, Gianluigi Arduini for his comments on the paper, and Ewen Maclean and Tobias Persson for discussion on the sources of lattice nonlinearities in the LHC.

*Sergey.Antipov@desy.de

[1] L. D. Landau, Zh. Eksp. Teor. Fiz. 16, 574 (1946); [J. Phys. (USSR) 10, 25 (1946)], https://doi.org/10.1016/B978-0-08010586-4.50066-3.
[2] J. H. Malmberg and C. B. Wharton, Phys. Rev. Lett. 13, 184 (1964).

[3] C. E. Nielsen, A. M. Sessler, and K. R. Symon, Longitudinal instabilities in intense relativistic beams, in Proceedings of HEACC'59 (CERN, Geneva, 1959.

[4] L. J. Laslett, V. K. Neil, and A. M. Sessler, Rev. Sci. Instrum. 36, 436 (1965).

[5] O. S. Brüning, P. Collier, P. Lebrun, S. Myers, R. Ostojic, J. Poole, and P. Proudlock, Report No. CERN-2004-003-V-1.

[6] J. Gareyte, J.P. Koutchouk, and F. Ruggiero, Report No. CERN-LHC-PROJECT-REPORT-091.

[7] G. Apollinari, I. Béjar Alonso, O. Brüning, M. Lamont, and L. Rossi, Report No. CERN-2015-005.

[8] A. Abada et al. (FCC Collaboration), Eur. Phys. J. Special Topics 228, 755 (2019).

[9] V. Kornilov, O. Boine-Frankenheim, and V. Kapin, Coherent instability thresholds and dynamic aperture with octupoles and nonlinear space-charge in the SIS100 synchrotron, in Proceedings of IPAC'10, Kyoto, Japan, TUPD029 (JACoW, Geneva, 2010).

[10] FAIR Technical Design Report SIS100, GSI, Darmstadt, Germany, 2008.

[11] K. Hübner, A. G. Ruggiero, and V. G. Vaccaro, Report No. CERN-ISR-TH-RF-69-23, Geneva, Switzerland, 1969.

[12] J. S. Berg and F. Ruggiero, Report No. CERN-SL-AP-96071-AP, Geneva, Switzerland, 1996.

[13] A. W. Chao and M. Tigner, Handbook of Accelerator Physics and Engineering (World Scientific, Singapore, 2006).

[14] V. Kornilov, O. Boine-Frankenheim, W. Kaufmann, and P. Moritz, Report No. GSI-Acc-Note-2006-12-001.

[15] Y. Luo, W. Fischer, A. Marusic, and M. Minty, Measurement and simulation of betatron coupling beam transfer function in RHIC, Proceedings of IPAC2018, Vancouver, BC, Canada (JACoW, Geneva, Switzerland, 2018).

[16] C. Tambasco et al., First BTF measurements at the Large Hadron Collider, Proceeding of IPAC2016, Busan, Korea (JACoW, Geneva, Switzerland, 2016).

[17] T. Pieloni et al., MD 3292: Summary of BTF studies MD Block 3\&4, CERN, 2018, https://indico.cern.ch/event/ 776844.

[18] T. Himel, Annu. Rev. Nucl. Part. Sci. 47, 157 (1997).

[19] J. Byrd and W. Barry, AIP Conf. Proc. 395, 167 (1997).

[20] K. Harkay, T. Berenc, J. Byrd, R. Dowd, L. Emery, D. Teytelman, and U. Wienands, Characterizing the coupled bunch driving terms in a storage ring, Proceedings of IBIC2017, Grand Rapids, MI (JACoW, Geneva, Switzerland, 2018).

[21] D. Heins, R. D. Kohaupt, W. Kriens, M. Leneke, K. H. Matthiesen, H. Musfeldt, and S. Patzold, Z. Phys. C 37, 165 (1987).

[22] R. Bartolini, R. Fielder, E. Koukovini-Platia, and G. Rehm, Multi-bunch instabilities measurement and analysis at the Diamond light source, Proceedings of IPAC2017, Copenhagen, Denmark (JACoW, Geneva, Switzerland, 2017).

[23] N. Eddy, B. Fellenz, P. Prieto, and S. Zorzetti, Transverse damper using diodes for slip stacking in the Fermilab Recycler, Proceedings of IBIC2017, Grand Rapids, MI (JACoW, Geneva, Switzerland, 2018). 
[24] Note that not all the coherent modes can be efficiently damped by a transverse feedback, in particular, those that create a small centroid offset but large intrabunch oscillations, hence the need of Landau damping for such modes.

[25] A. W. Chao, Physics of Collective Beam Instabilities in High-Energy Accelerators (Wiley, New York, 1993).

[26] A. Burov, Phys. Rev. Accel. Beams 17, 021007 (2014).

[27] S. Antipov et al., J. Instrum. 12, T03002 (2017).

[28] E. Stern, J. Amundson, and A. Macridin, Suppression of instabilities generated by an anti-damper with a nonlinear magnetic element in IOTA, Proceedings of IPAC2018, Vancouver, BC, Canada (JACoW, Geneva, Switzerland, 2018).

[29] A. Butterworth et al., LHC transverse feedback, in Proceedings of EVIAN'15, Evian-les-Bains, France (CERN, Geneva, 2015).

[30] G. Kotzian, W. Höfle, and D. Valuch, Sensitivity of the LHC transverse feedback system to intra-bunch motion, Proceedings of IPAC2017, Copenhagen, Denmark (JACoW, Geneva, Switzerland, 2017).

[31] M. Söderén and D. Valuch, EPJ Web Conf. 245, 01036 (2020).

[32] L. N. Drosdal, K. Cornelis, V. Kain, E. Veyrunes, W. Bartmann, C. Bracco, B. Goddard, and M. Meddahi, SPS transverse beam scraping and LHC injection losses, in Proceedings of IPAC'12, New Orleans, LA, TUPPR094 (JACoW, Geneva, 2012).

[33] See Supplemental Material at http://link.aps.org/supplemental/ 10.1103/PhysRevLett.126.164801 for an example of the gathered data.
[34] G. Kotzian, Transverse feedback parameter extraction from excitation data, Proceedings of IPAC2017, Copenhagen, Denmark (JACoW, Geneva, Switzerland, 2017).

[35] K. Li et al., Code development for collective effects, Proceedings of HB2016, Malmö, Sweden (JACoW, Geneva, Switzerland, 2016).

[36] E. Métral et al., IEEE Trans. Nucl. Sci. 63, 1001 (2016).

[37] X. Buffat et al., Summary of instability observations at LHC and implications for HL-LHC, CERN, 2019, https://indico .cern.ch/event/831847/.

[38] M. McAteer et al., Report No. CERN-ACC-NOTE-2014-0012.

[39] E. H. Maclean, Observations relating to MCDO alignment, CERN, https://indico.cern.ch/event/812944/.

[40] E. H. Maclean, R. Tomás, F. Schmidt, and T. H. B. Persson, Phys. Rev. Accel. Beams 17, 081002 (2014).

[41] E. H. Maclean, F. Carlier, M. Giovannozzi, T. Persson, and R. Tomás, Effect of linear coupling on nonlinear observables at the LHC, Proceedings of IPAC2017, Copenhagen, Denmark (JACoW, Geneva, Switzerland, 2017).

[42] L. R. Carver, X. Buffat, K. Li, E. Métral, and M. Schenk, Phys. Rev. Accel. Beams 21, 044401 (2018).

[43] D. Möhl, Part. Accel. 50, 177 (1995), https://cds.cern.ch/ record/280706/files/p177.pdf.

[44] E. Métral and F. Ruggiero, Report No. CERN-AB-2004025-ABP.

[45] K. Y. Ng, Report No. FERMILAB-CONF-08-410-AD.

[46] V. Kornilov, O. Boine-Frankenheim, and I. Hofmann, Phys. Rev. Accel. Beams 11, 014201 (2008). 